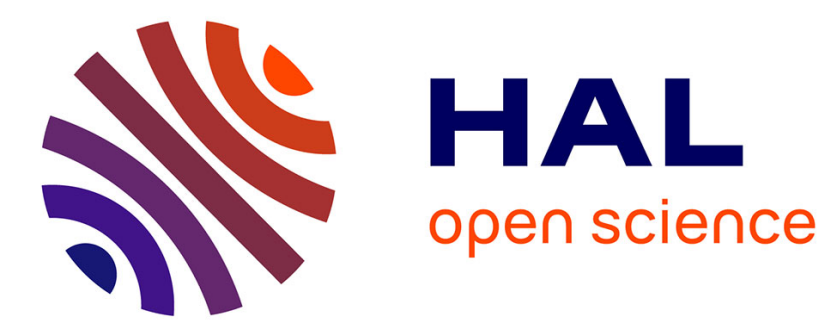

\title{
Etude d'un moteur à réluctance variable à disques imbriqués alimenté à tension constante
}

\author{
E. Salame, G. Goyet, J. Lucidarme, G. Quichaud, C. Rioux
}

\section{To cite this version:}

E. Salame, G. Goyet, J. Lucidarme, G. Quichaud, C. Rioux. Etude d'un moteur à réluctance variable à disques imbriqués alimenté à tension constante. Revue de Physique Appliquée, 1984, 19 (7), pp.533544. 10.1051/rphysap:01984001907053300 . jpa-00245223

\section{HAL Id: jpa-00245223 https://hal.science/jpa-00245223}

Submitted on 1 Jan 1984

HAL is a multi-disciplinary open access archive for the deposit and dissemination of scientific research documents, whether they are published or not. The documents may come from teaching and research institutions in France or abroad, or from public or private research centers.
L'archive ouverte pluridisciplinaire HAL, est destinée au dépôt et à la diffusion de documents scientifiques de niveau recherche, publiés ou non, émanant des établissements d'enseignement et de recherche français ou étrangers, des laboratoires publics ou privés. 


\title{
Etude d'un moteur à réluctance variable à disques imbriqués alimenté à tension constante
}

\author{
E. Salame, G. Goyet, J. Lucidarme, G. Quichaud et C. Rioux \\ Laboratoire d'Electrotechnique, Universités Paris 6 et 11, Bâtiment 214, 91405 Orsay, France \\ (Reçu le 11 janvier 1984, révisé le 21 mars, accepté le 23 mars 1984)
}

\begin{abstract}
Résumé. - Dans cet article, nous présentons une étude théorique et expérimentale d'un moteur à réluctance variable à disques imbriqués alimenté directement à $50 \mathrm{~Hz}$ par un réseau triphasé industriel à tension efficace constante. Les deux principales hypothèses faites dans ce travail sont les suivantes : les circuits magnétiques de la machine à réluctance variable sont non saturés et la ligne d'arbre entraînée est supposée infiniment rigide. Dans ce cadre, nous présentons les caractéristiques des divers fonctionnements synchrones possibles ainsi que leurs conditions de stabilité.
\end{abstract}

\begin{abstract}
Theory and experiment of a three phase reluctance motor, directly fed from a $50 \mathrm{~Hz}$ sine wave voltage, is presented. The motor is made of imbricate stator and rotor disks with numerous magnetic poles. The present paper utilizes two main hypothesis : magnetic circuits and poles work with no saturation and all parts of the mechanical shafting are supposed to be infinitly rigid. The analysis gives the characteristics of different synchronous possible drives and the corresponding stability conditions.
\end{abstract}

\section{Principe d'une machine à réluctance variable à disques imbriqués $[1,2,10]$.}

La figure 1 donne le principe d'une machine à réluctance variable à disques imbriqués et à alimentation monophasée. Une telle machine est constituée de disques mobiles isolants solidaires d'un même arbre et portant $p$ plots magnétiques régulièrement répartis. La rotation de ces disques devant des disques fixes identiques produit une variation de réluctance dans des bobinages de type solénoïdes entourant les disques. Une partie de ces bobinages est alimentée à partir d'un réseau alternatif à tension efficace constante alors qu'une autre partie, dans le cas d'une machine excitée, est alimentée à partir d'un courant continu d'intensité constante.

L'intérêt d'une telle structure réside dans la possibilité de couples volumiques élevés grâce à l'augmentation du nombre de disques fixes et mobiles par unité de longueur axiale de machine.

En groupant sur un même arbre trois machines monophasées dont les trois ensembles de disques sont décalés les uns par rapport aux autres d'un angle $\frac{2 \pi}{3 p}$ $\left(\frac{1}{3}\right.$ du pas des plots $)$ on obtient une machine à alimen-

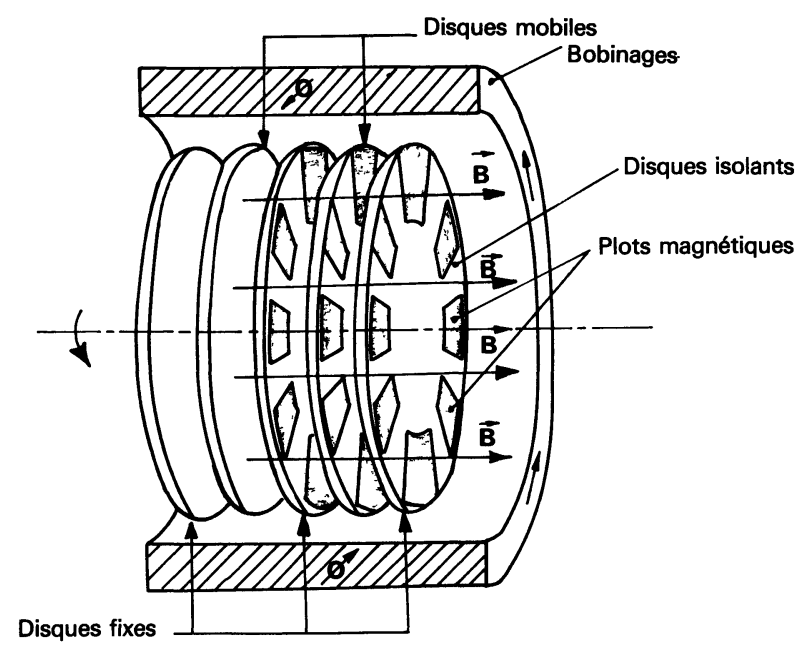

Fig. 1. - Schéma de principe d'une machine à réluctance variable.

[Principle of a reluctance motor.]

tation triphasée. Le prototype qui est l'objet de l'étude suivante est de ce type, chaque module étant constitué par deux disques mobiles et trois disques fixes. 
2. Modélisation d'une machine à réluctance variable triphasée [3-6].

Une machine à réluctance variable à alimentation triphasée étant constituée par trois modules semblables découplés au point de vue magnétique, sa modélisation se déduit de celle d'un seul de ses modules. La figure 2 représente un schéma de principe simplifié d'un module à partir duquel nous allons élaborer sa modélisation dans l'hypothèse d'un circuit magnétique non saturé et d'un couplage parfait entre bobinage d'alimentation alternatif et d'excitation continue.

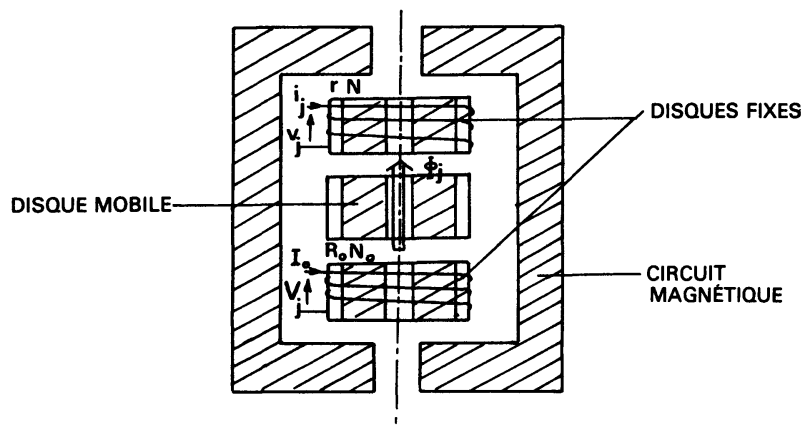

Fig. 2. - Schéma de principe d'un module.

[Simplified representation of a module.]

Nous obtenons ainsi pour chaque module les deux équations électriques suivantes dans l'hypothèse où les trois bobinages d'excitation sont parcourus par un même courant continu $I_{0}$, qui sera supposé constant dans la suite :

$$
\begin{aligned}
& v_{j}=r i_{j}+N \frac{\mathrm{d} \phi_{j}}{\mathrm{~d} t} \\
& V_{j}=R_{0} I_{0}+N_{0} \frac{\mathrm{d} \phi_{j}}{\mathrm{~d} t}
\end{aligned}
$$

$r$ et $N$ étant la résistance et le nombre de spires du bobinage destiné à être alimenté en alternatif et $R_{0}$ et $N_{0}$ la résistance et le nombre de spires du bobinage d'excitation. A ces équations électriques nous devons ajouter les trois équations magnétiques suivantes :

$$
N i_{j}+N_{0} I_{0}=R_{j}(\theta) \phi_{j}
$$

telles que :

$$
R_{j}(\theta)=R\left(\theta-(j-1) \frac{2 \pi}{3}\right)
$$

où la réluctance $R$ dépend de la position électrique angulaire $\theta$ des plots reliée à leur position angulaire mécanique $\theta_{\mathrm{m}}$ par la relation $\theta=p \theta_{\mathrm{m}}$ où $p$ représente le nombre de plots d'un disque.
L'énergie magnétique du système ainsi décrit se met sous la forme suivante :

$$
W_{\mathrm{M}}=\frac{1}{2} \sum_{j=1}^{3} R_{j}(\theta) \phi_{j}^{2}
$$

d'où nous déduisons l'expression du couple développé par la machine à réluctance :

$$
\Gamma=-\left(\frac{\partial W_{\mathrm{M}}}{\partial \theta_{\mathrm{m}}}\right)_{\phi_{j}=\mathrm{cte}}=-\frac{p}{2} \sum_{j=1}^{3} \phi_{j}^{2} \frac{\mathrm{d} R_{j}}{\mathrm{~d} \theta} .
$$

L'utilisation de cette méthode nécessite la connaissance des divers paramètres définissant un module soit :

$$
\begin{aligned}
p & =24 & & \\
R_{0} & =11 \mathrm{~m} \Omega & & N_{0}=14 \\
r & =22 \mathrm{~m} \Omega & & N=24 .
\end{aligned}
$$

Quant à la réluctance $R(\theta)$ d'un module, nous l'avons obtenue à partir d'un relevé de la tension à vide délivrée par la machine en rotation uniforme à la vitesse angulaire $\boldsymbol{\Omega}$ :

$$
\left(v_{1}\right)_{i_{1}=0}=p \Omega N N_{0} I_{0} \cdot \frac{\mathrm{d}\left(\frac{1}{R(\theta)}\right)}{\mathrm{d} \theta} .
$$

La figure 3 donne le relevé oscillographique de cette tension à $125 \mathrm{t} / \mathrm{min}$ pour $I_{0}=200 \mathrm{~A}$. Ainsi $R(\theta)$ est obtenue à une constante près par intégration de

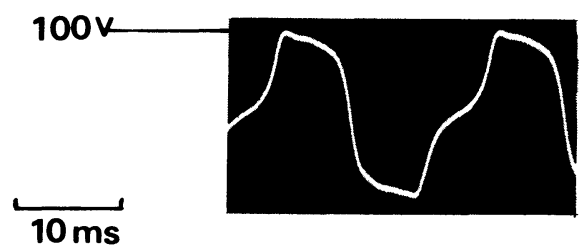

Fig. 3. - Tension à vide $\left(I_{0}=200 \mathrm{~A}-125 \mathrm{t} / \mathrm{min}\right)$.

[No load voltage $\left(I_{0}=200\right.$ A, 125 RPM $)$.]

cette tension à vide. La constante est déterminée grâce à (2) à partir du relevé (Fig. 4) du flux dans une spire des bobinages en fonction des ampères-tours, les plots étant en opposition $(\theta=0)$ ou en conjonction $(\theta=\pi)$. Cette figure précise également le domaine de validité des régimes linéaires que nous étudierons dans la suite.

Sur la figure 5 nous avons porté les variations de la réluctance et de sa dérivée $\frac{\mathrm{d} R}{\mathrm{~d} \theta_{\mathrm{m}}}$ en fonction de l'angle mécanique $\theta_{\mathrm{m}}$. Dans la suite nous utiliserons $R(\theta)$ sous la forme d'une série de Fourier tronquée telle que :

$$
\begin{aligned}
R(\theta) & =r_{0}+r_{1} \cos \theta+r_{2} \cos 2 \theta+r_{4} \cos 4 \theta \\
& =\sum_{n \geqslant 0} r_{n} \cos n \theta
\end{aligned}
$$




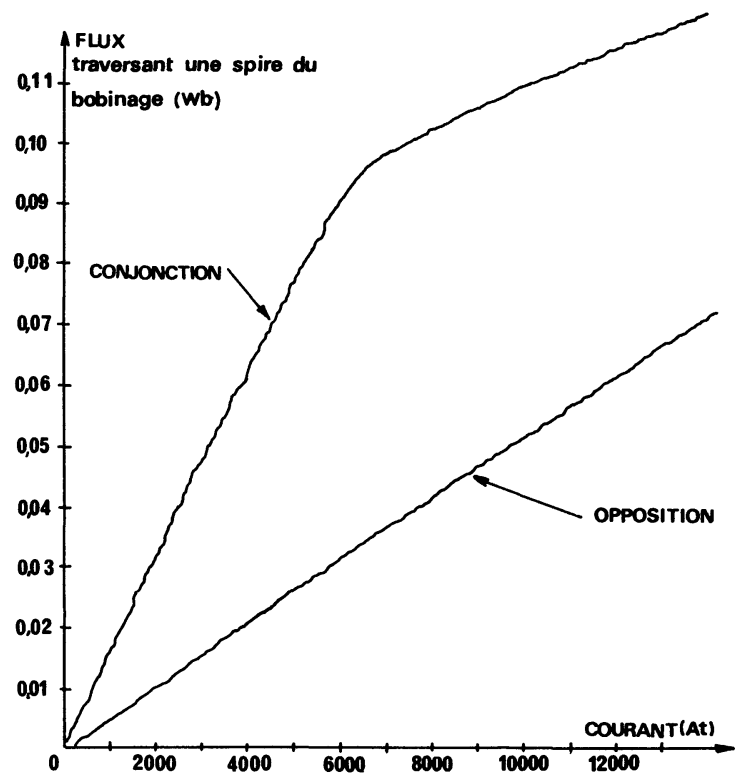

Fig. 4. - Flux dans une spire en fonction des ampèrestours.

[Flux-ampere turn-curve.]

où :

$$
\begin{aligned}
r_{0}= & 124449 \\
r_{1}= & 63341 \\
2 r_{2}= & 23880 \\
4 r_{4}= & -12500
\end{aligned}
$$

sont exprimés en ampère par weber.

\section{Fonctionnements synchrones [4].}

La machine à réluctance variable étant supposée alimentée par un système triphasé équilibré de tensions :

$$
v_{j}=V \sqrt{2} \sin \left(\omega t-(j-1) \frac{2 \pi}{3}\right)
$$

les chutes de tension $r i_{j}$ étant supposées faibles les flux $\phi_{j}$ sont pratiquement imposés à des constantes $K_{j}$ près soit :

$$
\Phi_{j}=-\Phi \cos \left(\omega t-(j-1) \frac{2 \pi}{3}\right)+K_{j}
$$
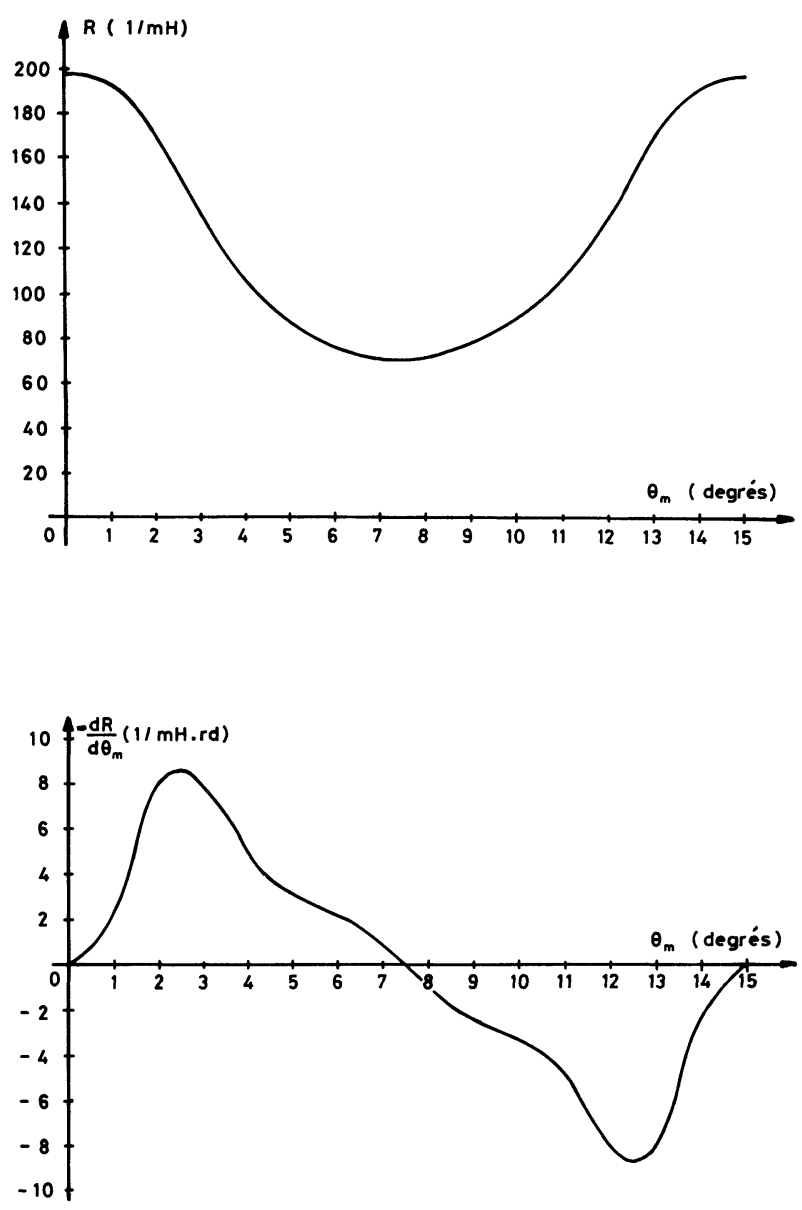

Fig. 5. - Variation de la réluctance et de sa dérivée en fonction de $\theta_{\mathrm{m}}$.

[Reluctance and its derived function in terms of angle of revolution $\theta_{\mathrm{m}}$.]

où :

$$
\phi=\frac{V \sqrt{2}}{N \omega} .
$$

Dans ces conditions, ce flux étant porté dans l'expression (5) et la réluctance étant prise sous la forme d'une série de Fourier nous obtenons pour expression du couple après linéarisation des produits de fonctions sinusoïdales :

$$
\begin{aligned}
\Gamma= & -\frac{p}{2}\left\{\sum _ { n \neq 3 N ^ { \prime } } n r _ { n } \left(3 \Phi K_{\mathrm{m}} \sin (n \theta \mp \omega t)+\Phi K_{\mathrm{s}} \sin (n \theta \pm \omega t \mp \delta)\right.\right. \\
& \left.-\frac{3}{4} \Phi^{2} \sin (n \theta \pm 2 \omega t)-K_{\mathrm{s} 2} \sin \left(n \theta \pm \delta_{2}\right)\right) \\
& \left.+\sum_{n=3 N^{\prime}} n r_{n}\left(\Phi K_{\mathrm{s}} \sin (n \theta-\omega t+\delta)+\Phi K_{\mathrm{s}} \sin (n \theta+\omega t-\delta)-3\left(K_{\mathrm{m} 2}+\frac{\Phi^{2}}{2}\right) \sin n \theta\right)\right\} .
\end{aligned}
$$


Dans ces sommes étendues aux divers ordres $n$ des harmoniques de réluctance figurent les signes \pm et nous devons prendre le signe supérieur $\sin =3 N^{\prime}+1$ et le signe inférieur si $n=3 N^{\prime}-1$ et les $\left(K_{\mathrm{m}}, K_{\mathrm{s}}, \delta\right)$ et $\left(K_{\mathrm{m} 2}, K_{\mathrm{s} 2}, \delta_{2}\right)$ sont calculés à partir des composantes de Clarke de $\left(K_{1}, K_{2}, K_{3}\right)$ et de $\left(K_{1}^{2}, K_{2}^{2}, K_{3}^{2}\right)$ comme nous allons l'indiquer.

Ainsi, si nous posons dans le cas général des trois constantes $\left(K_{1}, K_{2}, K_{3}\right)$ :

$$
\begin{array}{r}
\frac{1}{3}\left(K_{1}+K_{2}+K_{3}\right)=K_{\mathrm{m}} \\
K_{1} \sin \omega t+K_{2} \sin \left(\omega t-\frac{2 \pi}{3}\right)+K_{3} \sin \left(\omega t-\frac{4 \pi}{3}\right) \\
=K_{\mathrm{s}} \sin (\omega t+\delta) \\
K_{1} \sin \omega t+K_{2} \sin \left(\omega t-\frac{4 \pi}{3}\right)+K_{3} \sin \left(\omega t-\frac{2 \pi}{3}\right) \\
=K_{\mathrm{s}} \sin (\omega t-\delta),
\end{array}
$$

nous pouvons remarquer que $K_{\mathrm{m}}, K_{\mathrm{s}}$ et $\delta$ se calculent à partir de composantes du type Clarke telles que :

$$
\begin{gathered}
\left(\begin{array}{ccc}
\frac{1}{3} & \frac{1}{3} & \frac{1}{3} \\
1 & -\frac{1}{2} & -\frac{1}{2} \\
0 & -\frac{\sqrt{3}}{2} & \frac{\sqrt{3}}{2}
\end{array}\right) \times\left(\begin{array}{c}
K_{1} \\
K_{2} \\
K_{3}
\end{array}\right)=\left(\begin{array}{c}
K_{\mathrm{m}} \\
K_{\mathrm{d}} \\
K_{\mathrm{q}}
\end{array}\right) \\
K_{\mathrm{s}}^{2}=K_{\mathrm{q}}^{2}+K_{\mathrm{d}}^{2} \\
\operatorname{tg} \delta=\frac{K_{\mathrm{q}}}{K_{\mathrm{d}}}
\end{gathered}
$$

et nous obtenons de même $K_{\mathrm{m} 2}, K_{\mathrm{s} 2}$ et $\delta_{2}$ à partir des composantes de Clarke de $K_{1}^{2}, K_{2}^{2}$ et $K_{3}^{2}$.

Ainsi le couple de la machine à réluctance se met sous la forme suivante :

$$
\Gamma=\sum_{n, k} \Gamma_{n, k} \sin \left(n \theta+k \omega t+\alpha_{n, k}\right)
$$

où chacun des termes de la somme ne dépendant que de la variable $n \theta+k \omega t$ sera nommé dans la suite " couple glissant ».

En rotation uniforme la valeur moyenne de $\Gamma$ est nulle sauf dans le cas où le rotor est animé d'une vitesse constante telle que :

$$
n_{0} \theta+k_{0} \omega t=\theta_{0}
$$

où le couple $\left(n_{0}, k_{0}\right)$ figure dans la suite $(n, k)$ définie par (10). $k_{0}$ ne peut donc prendre que les valeurs \pm 1 ou $\pm 2, n_{0}$ étant un numéro d'harmonique de réluctance. Dans ces conditions, le couple développé par la machine à réluctance s'écrit :

$$
\Gamma=\hat{\Gamma}+\gamma(t)
$$

où $\hat{\Gamma}$ est un couple moyen :

$$
\hat{\Gamma}=\sum_{n, k} \Gamma_{n, k} \sin \left(\frac{n}{n_{0}} \theta_{0}+\alpha_{n, k}\right)
$$

pour lequel la somme le définissant est étendue à l'ensemble des $(n, k)$ compatibles avec (10) et vérifiant :

$$
n_{0} k=n k_{0}
$$

alors que le couple fluctuant $\gamma(t)$ s'écrit :

$$
\gamma(t)=\sum_{n, k} \Gamma_{n, k} \sin \left(\frac{n_{0} k-n k_{0}}{n_{0}} \omega t+\frac{n}{n_{0}} \theta_{0}+\alpha_{n, k}\right)
$$

où la somme est maintenant étendue aux valeurs complémentaires de $n$ et $k$.

Nous obtenons ainsi des fonctionnements synchrones en moteur ou générateur de la machine à réluctance variable dont les pulsations de synchronisme sont de la forme $\Omega_{0}=\frac{\omega}{p m_{0}}$ où $m_{0}=\frac{-n_{0}}{k_{0}}$. L'étude plus complète de ces régimes permanents synchrones nécessite le calcul des constantes d'intégration $K_{j}$ relatives à la détermination des flux. Pour cela on doit considérer ces régimes comme cas limites de régimes permanents périodiques dans lesquels les résistances sont faibles ce qui conduit à des courants $i_{j}$ de valeur moyenne nulle comme on le voit en prenant la valeur moyenne des deux membres de la première équation du système (1) dans l'hypothèse d'un flux borné. Ainsi pour les fonctionnements synchrones précédents pour lesquels $m_{0}=-\frac{n_{0}}{k_{0}}$ nous déterminons la constante $K_{j}$ en écrivant l'égalité des valeurs moyennes des deux membres de (2), soit :

où

$$
N_{0} I_{0}=r_{0}\left(K_{j}-K_{j}^{\prime}\right)
$$

$$
r_{0} K_{j}^{\prime}=\sum_{n \geqslant 1} r_{n} \widehat{\cos n \theta \cdot \phi} \cos \left(\omega t-(j-1) \frac{2 \pi}{3}\right)
$$

$K_{j}^{\prime}$ n'est différent de zéro que si $m_{0}$ est un entier positif ou négatif et prend les valeurs suivantes :

$$
K_{j}^{\prime}=\frac{1}{2} \phi \frac{r_{\left|m_{0}\right|}}{r_{0}} \cos \frac{\theta_{0}}{k_{0}}
$$

si $\left|m_{0}\right|=3 N^{\prime}+1$ et

$$
K_{j}^{\prime}=\frac{1}{2} \phi \frac{r_{\left|m_{0}\right|}}{r_{0}} \cos \left(\frac{\theta_{0}}{k_{0}} \pm(j-1) \frac{2 \pi}{3}\right)
$$

si $\left|m_{0}\right|=3 N^{\prime}-1$ (signe supérieur + ) ou $\left|m_{0}\right|=$ $3 N^{\prime}$ (signe inférieur -).

Ainsi le flux moyen dans la machine à réluctance variable se met sous la forme :

$$
\hat{\phi}_{i}=\frac{N_{0} I_{0}}{r_{0}}+K_{j}^{\prime}
$$


où l'origine des $K_{j}^{\prime}$ est dans un " redressement " des ampères-tours alternatifs absorbés, puisque le flux moyen $\phi_{j}$ dépend non seulement du courant continu $I_{0}$ mais également de l'amplitude $\phi$ du flux alternatif imposé.

Grâce à (2) nous pouvons également mettre le courant absorbé $i_{1}$ sous la forme suivante :

$$
\begin{aligned}
i_{1} & =\sum_{n \neq-m_{0}}-\frac{r_{n} \phi}{2 N} \cos \left[\left(\frac{n}{m_{0}}+1\right) \omega t+\frac{n}{n_{0}} \theta_{0}\right] \\
& +\sum_{n \neq m_{0}}-\frac{r_{n} \phi}{2 N} \cos \left[\left(\frac{n}{m_{0}}-1\right) \omega t+\frac{n}{n_{0}} \theta_{0}\right] \\
& +\sum_{n \neq 0} \frac{K_{1} r_{n}}{N} \cos \left(\frac{n}{m_{0}} \omega t+\frac{n}{n_{0}} \theta_{0}\right),
\end{aligned}
$$

dont le fondamental a pour expression :

$$
\begin{aligned}
i_{1 \mathrm{~F}}=-\frac{r_{0} \phi}{N} & \cos \omega t+\frac{K_{1} r_{\left|m_{0}\right|}}{N} \cos \left(\omega t+\frac{m_{0}}{n_{0}} \theta_{0}\right)- \\
& -\frac{r_{\left|2 m_{0}\right|} \phi}{2 N} \cos \left(\omega t+\frac{2 m_{0}}{n_{0}} \theta_{0}\right) .
\end{aligned}
$$

Le complément $\left(i_{\mathrm{F}}-i_{1 \mathrm{~F}}\right)$ étant constitué par des harmoniques ou également des sous-harmoniques si $n_{0}$ et $\left|m_{0}\right|$ sont différents de 1 .

Sur la figure 6 , nous avons porté le couple moyen ainsi que la valeur efficace du couple pulsé avec $\left(I_{0}=200 \mathrm{~A}\right)$ ou sans excitation pour les principaux fonctionnements synchrones possibles du prototype de machine à réluctance variable expérimenté.

\section{Etude expérimentale à la première vitesse de syn-} chronisme [4].

Comme le montre la figure 6, le fonctionnement synchrone le plus intéressant correspond à $n_{0}=1$ et $k_{0}=-1$ soit $m_{0}=1$, la pulsation de rotation correspondante étant alors $\frac{\omega}{p}$ soit pour une alimentation à $50 \mathrm{~Hz}$ une vitesse de rotation de $125 \mathrm{t} / \mathrm{min}$. Dans ces conditions, les trois flux moyens $K_{j}$ sont égaux à une valeur commune :

$$
K=\frac{N I_{0}}{r_{0}}+\frac{1}{2} \phi \frac{r_{1}}{r_{0}} \cos \theta_{0}
$$

et seules les composantes de Clarke $K_{\mathrm{m}}$ et $K_{\mathrm{m} 2}$ sont différentes de zéro. Dans l'hypothèse de la formule (7), où on ne considère en plus du fondamental que les harmoniques 2 et 4 de réluctance, on a alors les expressions suivantes pour le couple moyen $\hat{\Gamma}$ et le couple fluctuant $\gamma(t)$.

$$
\hat{\Gamma}\left(\theta_{0}\right)=-\frac{3 p}{2}\left(\phi K r_{1} \sin \theta_{0}-\frac{1}{2} \phi^{2} r_{2} \sin 2 \theta_{0}\right)
$$

$$
\begin{aligned}
& \gamma\left(\theta_{0}, t\right)=\frac{3 p}{2} \frac{\phi^{2}}{4} r_{1} \sin \left(3 \omega t+\theta_{0}\right)- \\
& \quad-2 r_{2} \phi K \sin \left(3 \omega t+2 \theta_{0}\right)-4 r_{4} \phi K \sin \left(4 \omega t+4 \theta_{0}\right) \\
& \quad+r_{4} \phi^{2} \sin \left(6 \omega t+4 \theta_{0}\right) .
\end{aligned}
$$

Quant au fondamental du courant absorbé par la

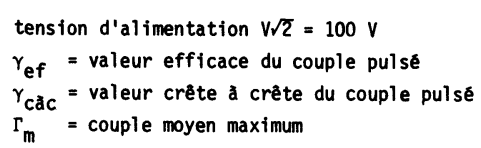


première phase son amplitude complexe $\bar{I}$ se met sous la forme suivante :

$$
\begin{aligned}
\bar{I}=\left[-j \frac{r_{0}}{N^{2} \omega}+j\right. & \frac{K r_{1}}{N V \sqrt{2}} \exp \left(j \theta_{0}\right)- \\
& \left.-j \frac{r_{2}}{2 N^{2} \omega} \exp \left(j 2 \theta_{0}\right)\right] \bar{V}
\end{aligned}
$$

où $\bar{V}$ représente l'amplitude complexe de la tension d'alimentation correspondante. Cette expression permet de tracer (Fig. 7) la courbe de description du courant en fonction du décalage angulaire $\theta_{0}$ qui est un cercle lorsqu'on néglige le flux redressé $\left(K_{j}^{\prime}=0\right)$ ainsi que les harmoniques de réluctance. La partie en trait plein de cette courbe correspond aux fonctionnements classiques stables de la machine synchrone tels que $\frac{\mathrm{d} \hat{\Gamma}}{\mathrm{d} \theta_{0}}<0$.

Afin de vérifier les résultats précédents, nous avons effectué des essais au moyen du montage indiqué sur la figure 8. La machine à réluctance variable excitée et alimentée par le réseau EDF est entraînée grâce au groupe Ward Léonard jusqu'à la vitesse de synchronisme désirée $(125 \mathrm{t} / \mathrm{min})$. On annule alors grâce à

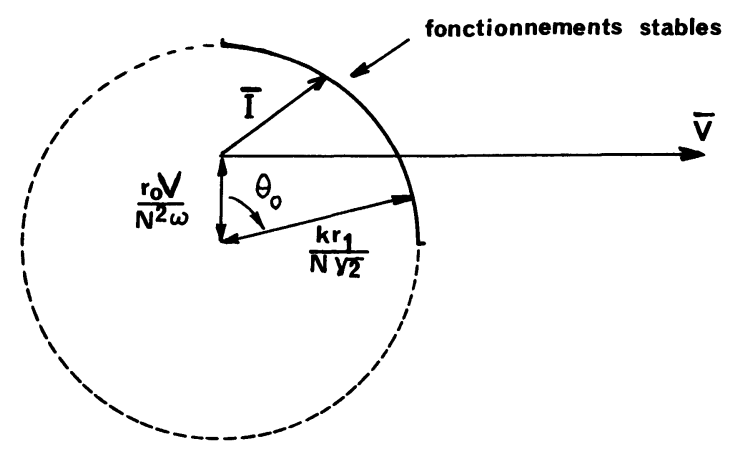

Fig. 7. - Courbe de description $\bar{I}\left(\theta_{0}\right)(125 \mathrm{t} / \mathrm{min})$.

[Description current diagram at 125 RPM.]

l'excitation réglable de la génératrice à courant continu le courant d'induit du moteur à courant continu d'entraînement, ce qui conduit à l'accrochage de la machine à réluctance sur le réseau et il suffit alors d'ouvrir l'alimentation du moteur à courant continu et de le faire fonctionner en génératrice sur résistance pour charger le moteur à réluctance variable.

Les résultats des essais sont portés sur les figures 9 à 12 . Ainsi sur la première figure 9 , nous avons tracé, à

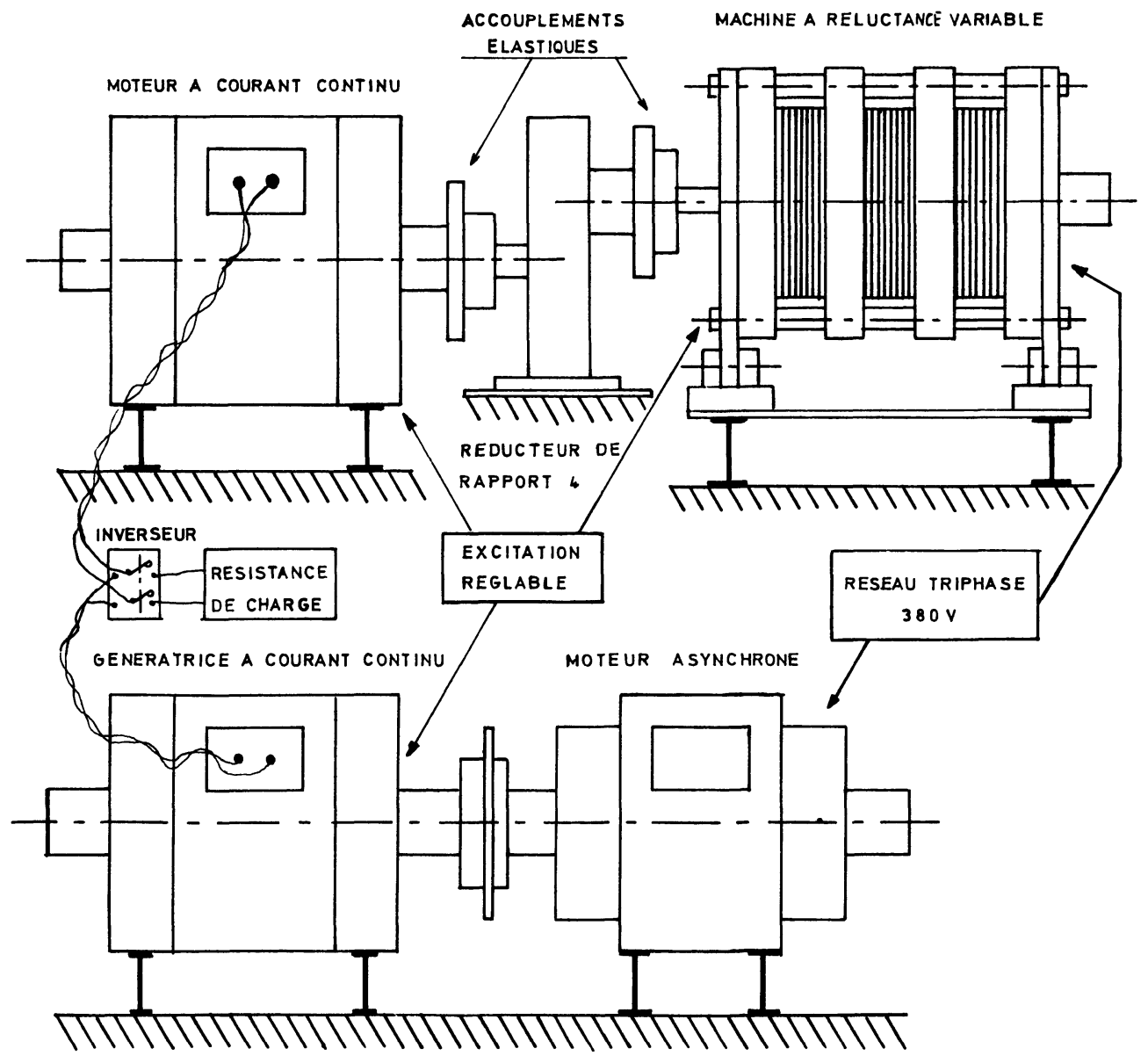

Fig. 8. - Schéma du montage d'essai.

[Test equipment.] 


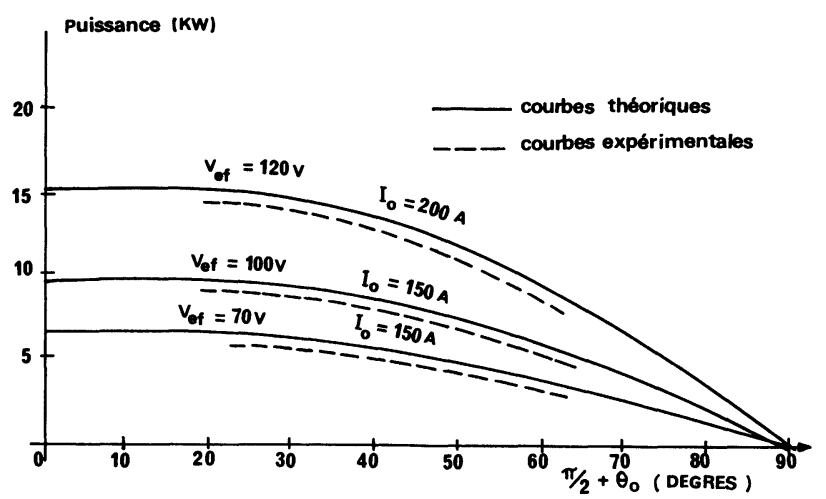

Fig. 9. - Puissance en fonction du décalage angulaire (125 t/min).

[Variation of the power with angle of phase difference at 125 RMP.]

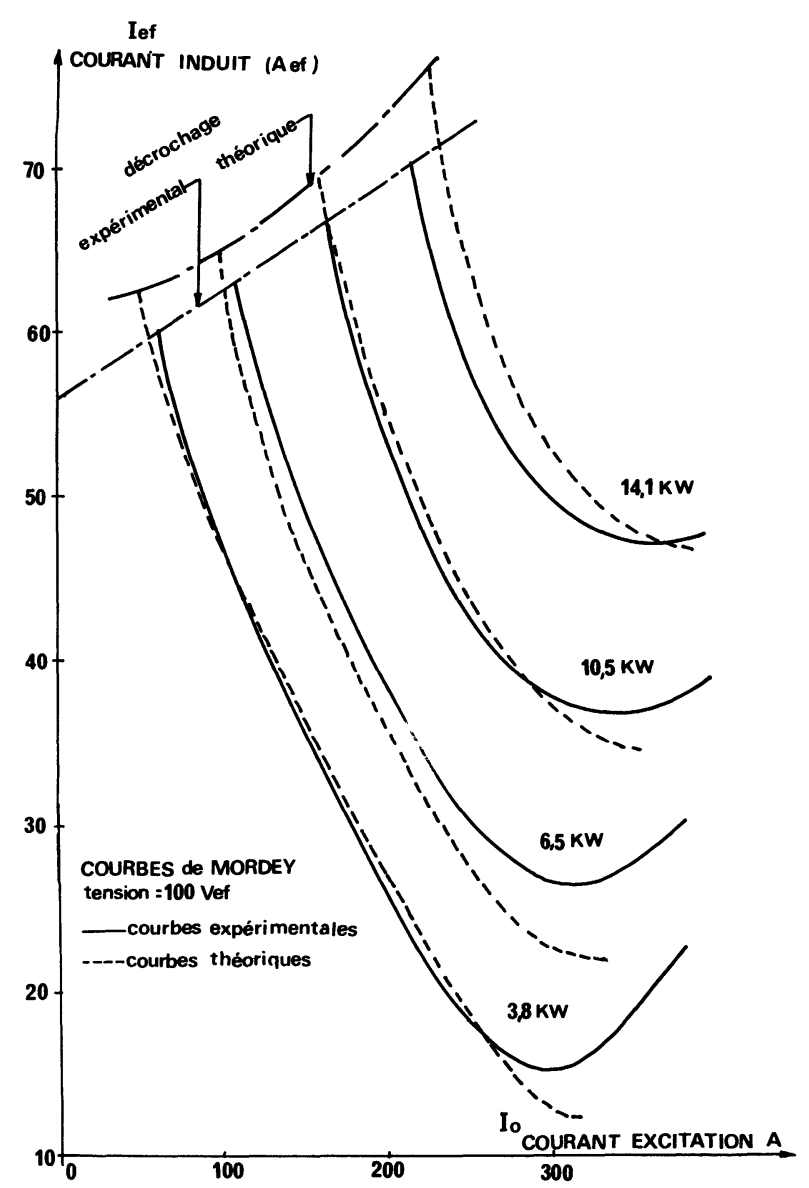

Fig. 10. - Courbes de Mordey (125 t/min).

[Mordey curves at 125 RPM.]

tension d'alimentation et excitation constante, la puissance en fonction du décalage angulaire $\theta_{0}$. L'allure de la courbe est bien caractéristique d'une machine synchrone de même que les courbes de Mordey que nous avons portées sur la figure 10. Sur les figures 11,12 , nous avons montré l'influence du courant d'excitation, à puissance délivrée constante,

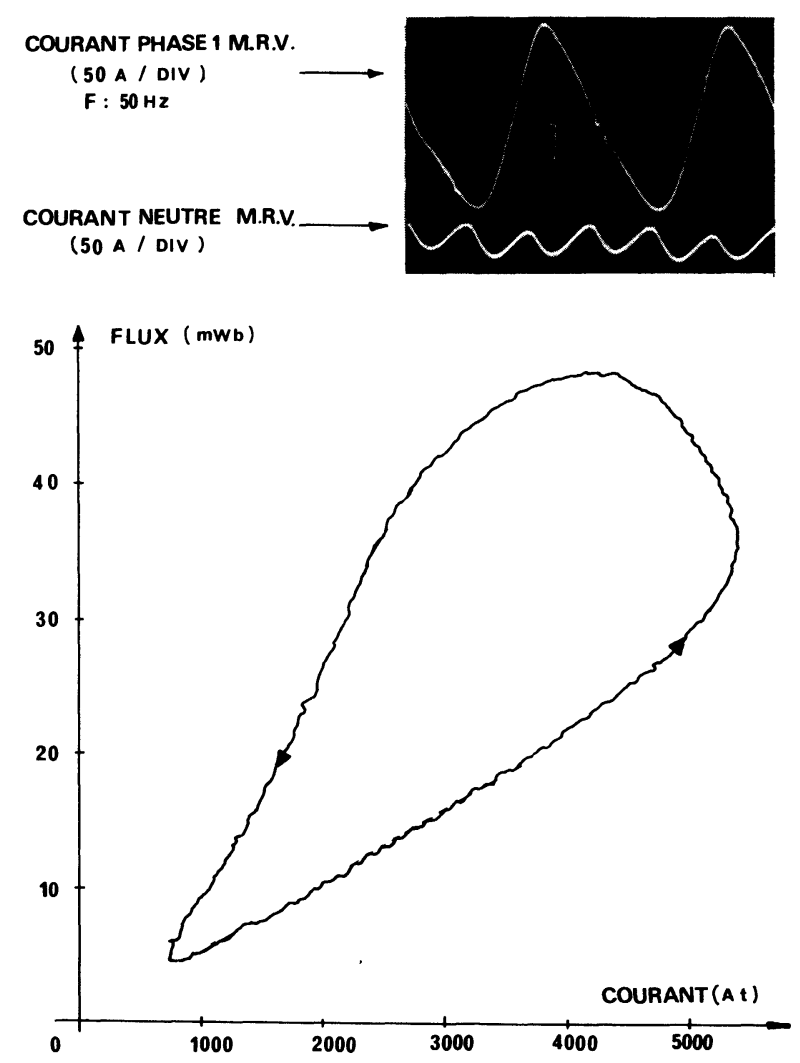

Fig. 11. - Oscillogramme des courants et cycle fluxampères-tours $\left(V=120 \mathrm{~V}, I_{0}=207 \mathrm{~A}, P=14 \mathrm{~kW}, 125 \mathrm{t} /\right.$ $\min )$.

[Current oscillogram and flux-ampere turn-cycles $(V=$ $\left.120 \mathrm{~V}, I_{0}=207 \mathrm{~A}, P=14 \mathrm{~kW}, 125 \mathrm{RPM}\right)$.]

sur le courant absorbé et sur la forme du cycle fluxampère-tours dont la surface est proportionnelle à la puissance fournie.

\section{Etude expérimentale à la deuxième vitesse de syn- chronisme [4].}

La figure 6 montre un deuxième fonctionnement synchrone possible pour lequel les couples pulsés sont tolérables. Ce fonctionnement s'effectue à une vitesse double de la précédente $(250 \mathrm{t} / \mathrm{min})$ et sans excitation. Ce fonctionnement utilise toujours le fondamental de la réluctance $\left(n_{0}=1\right)$ mais avec $k_{0}=2$ soit $m_{0}=-\frac{1}{2}$, ce qui conduit à des flux moyens $K_{j}$ nuls. Ainsi dans ce cas, avec les hypothèses précédentes concernant la réluctance, nous avons les expressions suivantes pour les couples moyen et pulsé :

$$
\begin{aligned}
& \hat{\Gamma}\left(\theta_{0}\right)=\frac{3 p}{8} \phi^{2} r_{1} \sin \theta_{0} \\
& \gamma\left(\theta_{0}, t\right)=-\frac{3 p}{4} \phi^{2} \times \\
& \times\left[r_{2} \sin \left(6 \omega t-2 \theta_{0}\right)+2 r_{4} \sin \left(6 \omega t-4 \theta_{0}\right)\right]
\end{aligned}
$$




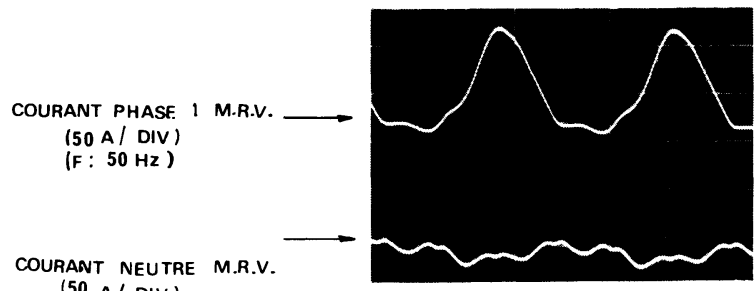

(50 A / DIV)

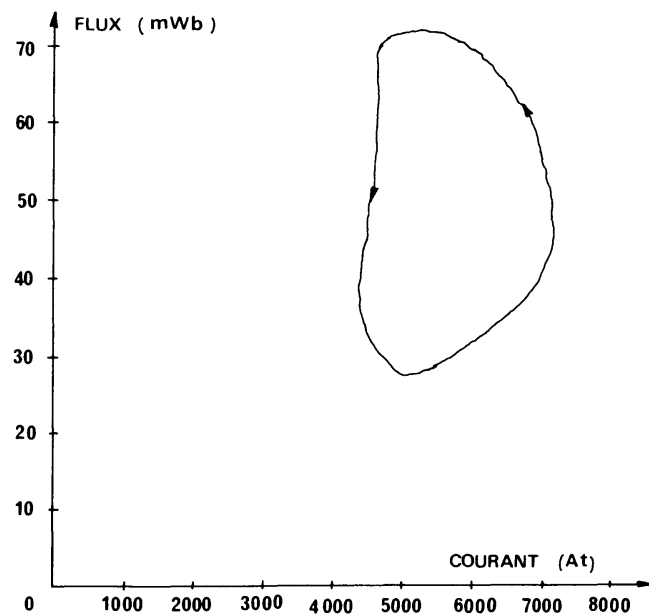

Fig. 12. - Oscillogramme des courants et cycle fluxampères-tours $\left(V=120 \mathrm{~V}, I_{0}=383 \mathrm{~A}, P=14 \mathrm{~kW}, 125 \mathrm{t} /\right.$ $\min )$.

[Current oscillogram and flux-ampere turn-cycles $(V=$ $\left.120 \mathrm{~V}, I_{0}=383 \mathrm{~A}, P=14 \mathrm{~kW}, 125 \mathrm{RPM}\right)$. $]$

ainsi que pour l'amplitude complexe du courant absorbé :

$$
\bar{I}=\left[-j \frac{r_{0}}{N^{2} \omega}-j \frac{r_{1}}{2 N^{2} \omega} \exp -j \theta_{0}\right] \bar{V}
$$

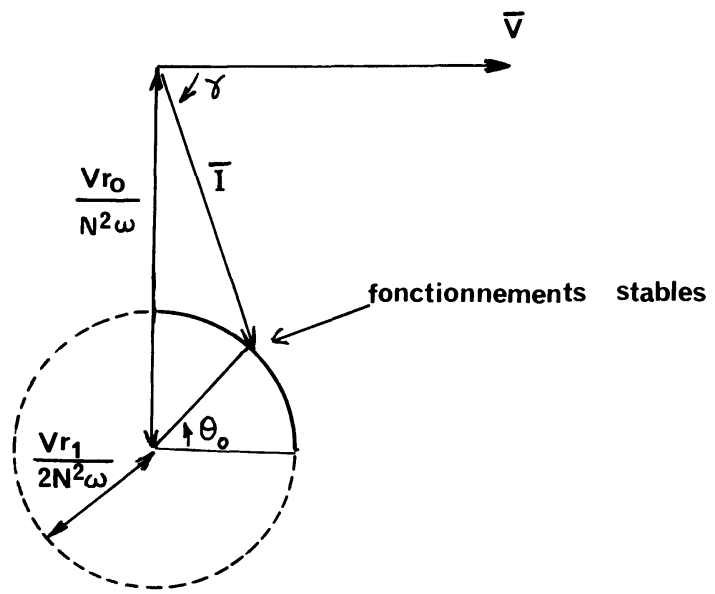

Fig. 13. - Courbe de description $I\left(\theta_{0}\right)(250 \mathrm{t} / \mathrm{min})$.

[Description current diagram at 125 RPM.]

dont la courbe de description circulaire est donnée sur la figure 13. Comme pour les essais précédents, nous avons porté (Fig. 14) la puissance en fonction du décalage angulaire à tension d'alimentation constante. L'absence de courant d'excitation ne permet évidemment plus d'avoir son influence sur le courant absorbé et le facteur de puissance. Notons (Fig. 15) que le facteur de puissance est toujours très faible, inférieur à 0,25 , dans ce deuxième fonctionnement alors que dans le premier il pouvait être proche de 1 . L'allure des courants ainsi que celle du flux en fonction des ampères-tours sont portées sur la figure 16 .

Notons au niveau de chaque phase la présence d'un harmonique 3 de courant important et qui se retrouve dans le neutre alors que dans le fonctionnement précédent avec excitation, les harmoniques 2 des courants de phase avaient une somme nulle dans le neutre comme le montre l'expression (21).

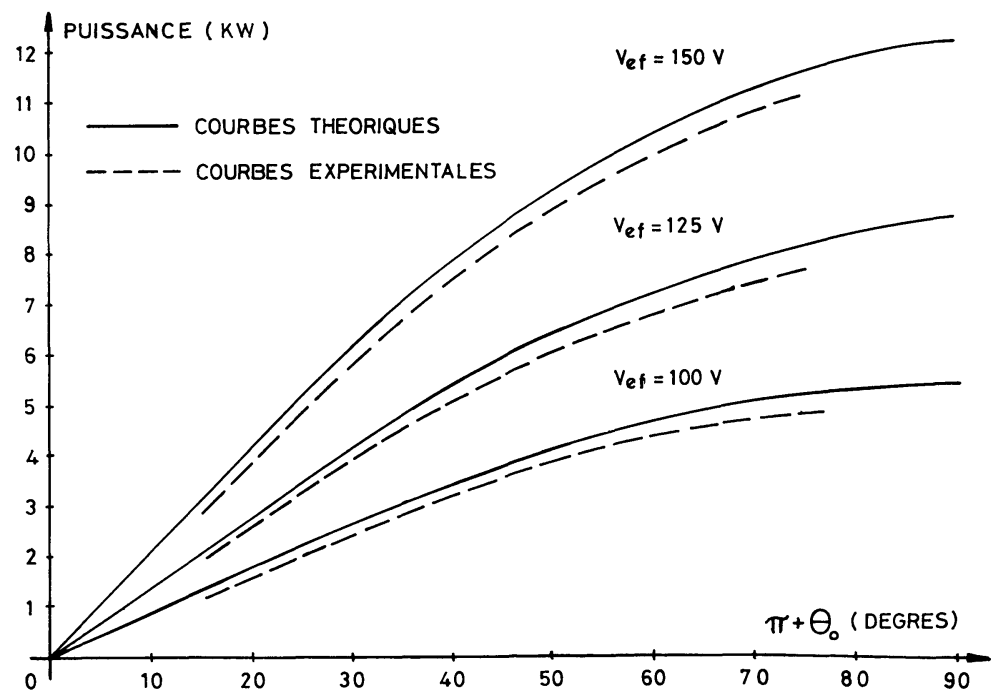

Fig. 14. - Puissance en fonction du décalage angulaire $(250 \mathrm{t} / \mathrm{min})$.

[Variation of the power with angle of phase difference at 250 RPM.] 


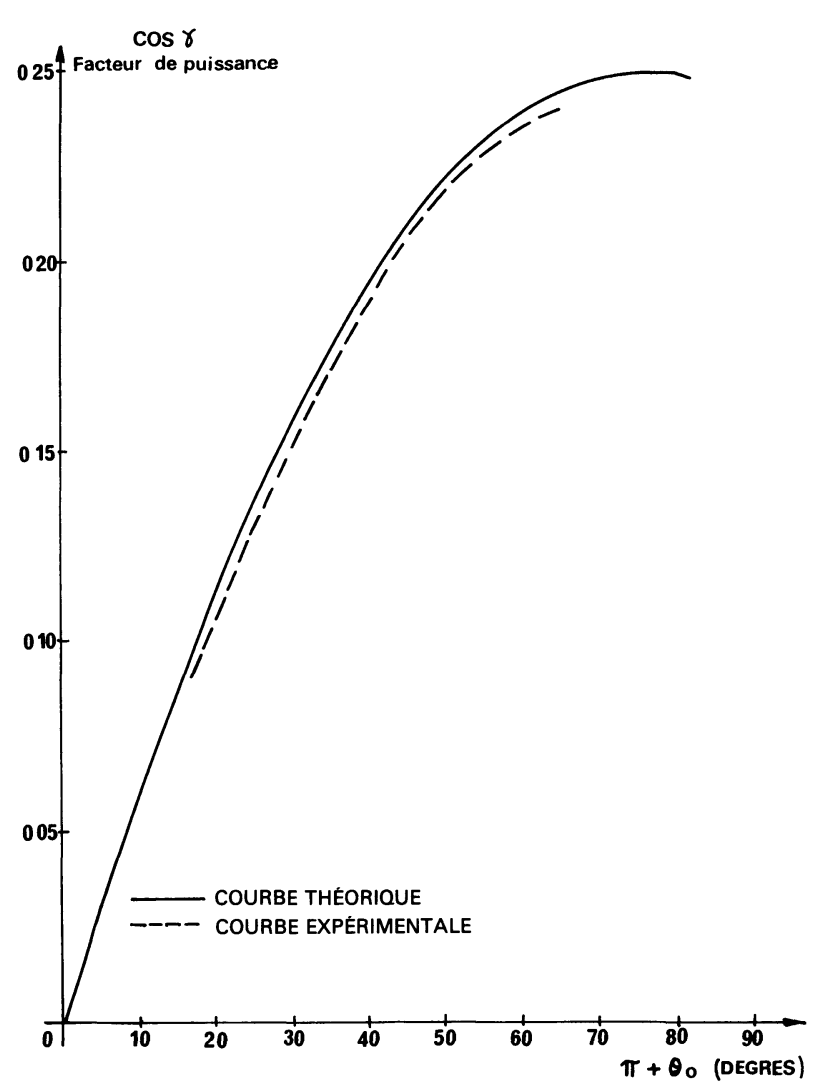

Fig. 15. - Facteur de puissance en fonction du décalage angulaire $(250 \mathrm{t} / \mathrm{min})$.

[Variation of the power factor with angle of phase difference at $250 \mathrm{RPM}$.]

\section{Stabilité des fonctionnements synchrones [4]}

Lorsque la machine synchrone entraîne une ligne d'arbre dont l'inertie $J$ est finie, des fluctuations de vitesse se produisent par rapport à la vitesse de synchronisme. Dans notre cas, l'équation mécanique s'écrit :

$$
\frac{J}{p} \frac{\mathrm{d}^{2} \theta}{\mathrm{d} t^{2}}=\Gamma-\Gamma_{0}-f \Omega
$$

où $\Gamma$ est le couple moteur que nous supposerons toujours imposé sous la forme (10) et $\Gamma_{0}+f \Omega\left(\Gamma_{0}\right.$ et $f$ constants) le couple de charge.

Ainsi, pour l'étude de la stabilité au voisinage de la première vitesse de synchronisme, nous effectuerons les changements de variables suivants :

$$
\begin{aligned}
\omega t & =t^{\prime} \\
\theta-\omega t & =\varphi\left(t^{\prime}\right)
\end{aligned}
$$

où $\varphi\left(t^{\prime}\right)$ joue maintenant le rôle d'un calage angulaire $\theta_{0}$ dépendant du temps et régit par l'équation différentielle du second ordre suivante :

$$
\frac{\mathrm{d}^{2} \varphi}{\mathrm{d} t^{\prime 2}}+a \frac{\mathrm{d} \varphi}{\mathrm{d} t^{\prime}}+\frac{\mathrm{d} G}{\mathrm{~d} \varphi}=h\left(\varphi, t^{\prime}\right)
$$
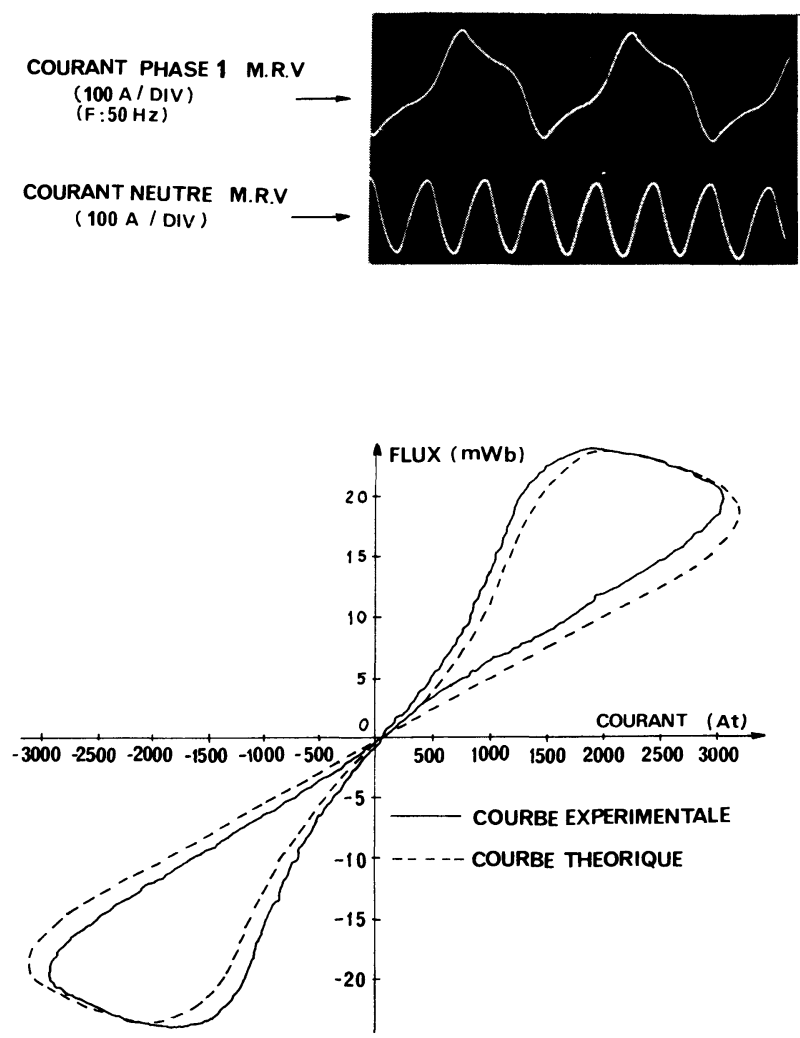

Fig. 16. - Oscillogramme des courants et cycle fluxampères-tours $(V=125 \mathrm{~V}, P=7,2 \mathrm{~kW}, \cos \gamma=0,24$, $250 \mathrm{t} / \mathrm{min})$.

[Current oscillogram and flux-ampere turn-cycles $(V=$ $125 \mathrm{~V}, P=7.2 \mathrm{~kW}, \cos \gamma=0.24,250 \mathrm{RPM})$.$] .$

où

$$
\begin{aligned}
a & =\frac{f}{J \omega} \\
G(\varphi) & =\frac{p}{J \omega^{2}} \int \hat{\Gamma}(\varphi) \mathrm{d} \varphi+F_{0} \varphi \\
F_{0} & =\frac{f}{J \omega}\left(1+\frac{p \Gamma_{0}}{f \omega}\right)
\end{aligned}
$$

et

$$
h\left(\varphi, t^{\prime}\right)=-\frac{p}{J^{2}} \gamma\left(\varphi, \frac{t^{\prime}}{\omega}\right)
$$

Les expressions de $\hat{\Gamma}$ et $\gamma$ sont respectivement données par (24) et (25). Ainsi (33) représente l'équation pendulaire d'une machine synchrone dans laquelle nous tenons compte des couples glissants parasites représentés par $f\left(\varphi, t^{\prime}\right)$ et où $\varphi$ et $g=-\frac{\mathrm{d} \varphi}{\mathrm{d} t^{\prime}}$ représentent respectivement le calage angulaire du rotor et son glissement $g=\frac{\omega-p \frac{\mathrm{d} \theta}{\mathrm{d} t}}{\omega}$

Dans le cas d'une machine synchrone classique les couples fluctuants $\gamma\left(\theta_{0}, t\right)$ sont faibles aussi on néglige 
le second membre de (33) et on considère l'équation pendulaire sous la forme simplifiée suivante :

$$
\frac{\mathrm{d}^{2} \varphi}{\mathrm{d} t^{\prime 2}}+a \frac{\mathrm{d} \varphi}{\mathrm{d} t}+\frac{\mathrm{d} G}{\mathrm{~d} \varphi}=0
$$

dont la stabilité s'étudie simplement au moyen de l'intégrale première suivante :

$$
\frac{1}{2} g^{2}+G(\varphi)=E_{0}-a \int_{t_{0}}^{t_{r}} g^{2} \mathrm{~d} t^{\prime}
$$

où la constante d'intégration $E_{0}$ est définie au moyen des conditions initiales $g_{0}, \varphi_{0}$ à l'instant $t_{0}$, soit :

$$
E_{0}=\frac{1}{2} g_{0}^{2}+G\left(\varphi_{0}\right)
$$

L'intégrale figurant au second membre de (39) étant toujours positive une condition suffisante de stabilité est que le système non amorti $(a=0)$ soit stable. Or pour ce dernier, l'étude est simplifiée par le fait que la trajectoire dans le plan de phase a pour équation :

$$
\frac{\mathrm{d} \varphi}{\mathrm{d} t}= \pm \sqrt{2 E_{0}-2 G(\varphi)}
$$

Ainsi, une condition suffisante de stabilité est que la position initiale $\varphi_{0}$ soit encadrée par deux racines $\varphi_{1}, \varphi_{2}$ de $E_{0}-G(\varphi)$ et on a alors une oscillation de $\varphi$ entre $\varphi_{1}$ et $\varphi_{2}$, oscillation amortie le cas échéant si $a^{\prime} \neq 0$.

Dans le cas où les couples glissants parasites ne sont plus négligés, il apparaît au second membre de l'intégrale première précédente (39) l'expression suivante :

$$
\int_{\varphi_{0}}^{\varphi} h\left(\varphi, t^{\prime}\right) \mathrm{d} \varphi
$$

qui est proportionnelle à l'énergie transférée au rotor par le couple parasite $\gamma\left(\varphi, \frac{t^{\prime}}{\omega}\right)$ donné par (25) et qui se présente sous la forme d'une somme de couples glissants :

$$
\gamma\left(\varphi, \frac{t^{\prime}}{\omega}\right)=\sum_{m, l} \gamma_{m, l} \sin \left(m \varphi+l t^{\prime}\right) .
$$

Nous allons voir dans la suite que ces couples glissants peuvent avoir une influence sur l'énergie du rotor et, en particulier, si l'inertie de la ligne d'arbre est suffisamment faible, conduire à une rotation chaotique.

\section{Influence des couples pulsés sur la stabilité de la machine à réluctance variable. Instabilité stochas- tique $[4,7-9]$.}

Nous présentons dans ce paragraphe une étude de la stabilité prenant en compte les couples parasites glissants qui nous a été suggérée par F. Escande [7].
Cette étude est basée sur l'analogie entre le mouvement du rotor de la machine à réluctance variable sous l'action des " couples glissants " définis par (11) et le mouvement d'une particule dans deux champs de force glissants pour lequel il apparaît des instabilités stochastiques qui sont un sujet d'intérêt actuel pour les physiciens, par exemple pour expliquer des phénomènes relatifs à la turbulence [9]. Notons d'ailleurs que des études expérimentales de ces instabilités s'effectuent au moyen de dispositifs qui ne sont rien d'autre que de très petits moteurs synchrones monophasés à vide constitués par une boussole placée dans un champ magnétique uniforme oscillant [8] et pour lequel un mouvement chaotique de l'aiguille aimantée apparaît pour certaines valeurs d'un paramètre du système appelé paramètre de stochasticité $s$. Ainsi si $s$ dépasse un certain seuil $s_{0}$ appelé seuil de stochasticité à grande échelle, le mouvement de la boussole est très désordonné ainsi par exemple, ayant accroché un champ tournant, elle pourra s'écarter assez rapidement de cette vitesse de synchronisme et s'accrocher sur l'autre champ tournant dont la vitesse est opposée, un mouvement intermédiaire pouvant être une vibration autour de la direction du champ magnétique stationnaire.

Le seuil de stochasticité $s_{0}$ à grande échelle est connue [7] dans le cas de l'équation suivante relative au mouvement d'une particule dans deux champs de forces glissants

$$
\frac{\mathrm{d}^{2} y}{\mathrm{~d} x^{2}}+A \sin y+B \beta \sin \beta(y-x)=0 .
$$

Le paramètre de stochasticité étant ainsi défini :

$$
s=2 \sqrt{A}+2 \sqrt{B}
$$

les instabilités stochastiques à grande échelle apparaissent lorsque $s \geqslant s_{0}$. Les variations de ce seuil $s_{0}$, en fonction de $\beta$ pour $\rho=\sqrt{\frac{A}{B}}=1$ et de $\rho=\sqrt{\frac{A}{B}}$ pour $\beta=1$ nous ont été données par F. Escande [7] (Fig. 17).
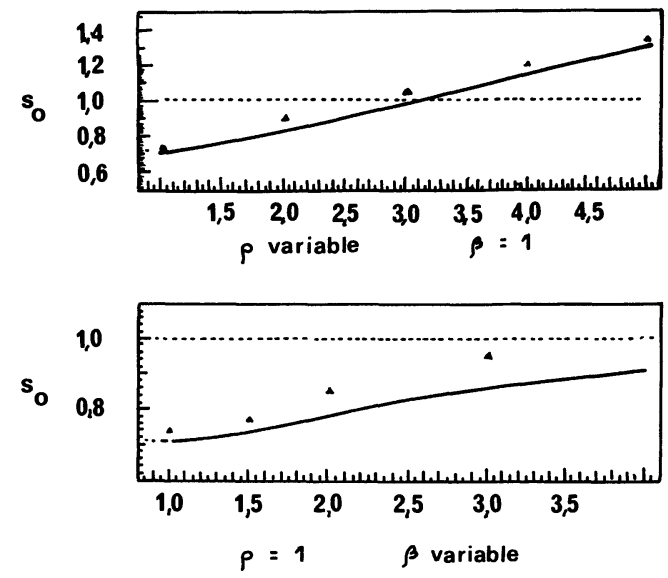

Fig. 17. - Variations du seuil de stochasticité [7].

[Variations of stochastic level [7].] 
Dans le cas où à la fois $\rho$ et $\beta$ sont différents de un, nous pouvons prendre [7] comme valeur approchée du seuil de stochasticité $s_{0}=0,7$.

Dans le cas où nous négligeons $a$ et $F_{0}$ et ne considérons que le fondamental $F \sin \varphi$ de la partie oscillante périodique de $\frac{\mathrm{d} G}{\mathrm{~d} \varphi}\left(F=\frac{3}{2} \frac{p^{2}}{J \omega^{2}} \frac{r_{1}}{r_{0}} N_{0} I_{0} \Phi\right)$ alors

s'écrit si nous nous limitons à un seul couple glissant parasite d'ordre $m, l$ :

$$
\frac{\mathrm{d}^{2} \varphi}{\mathrm{d} t^{\prime 2}}+F \sin \varphi=c \sin \left(m \varphi+l t^{\prime}\right)
$$

où ici :

$$
c=-\frac{p}{J \omega^{2}} \gamma_{m, l}
$$

Nous constatons qu'après avoir effectué le changement de variable $x=\frac{-l}{m} t^{\prime}$ et posé :

$$
\begin{aligned}
& \beta=m \\
& A=F \frac{m^{2}}{l^{2}} \\
& B=c \frac{m}{l^{2}}
\end{aligned}
$$

que cette équation (45) se ramène à (44), ce qui donne l'expression suivante du paramètre de stochasticité de la rotation de l'arbre de la machine à réluctance variable, soit :

$$
s=\frac{2 m}{l}\left(1+\sqrt{\frac{c}{m F}}\right) \frac{\omega_{0}}{\omega}
$$

où $\omega_{0}=\omega \sqrt{F}$ est la pulsation pendulaire de la ligne d'arbre non perturbée par le couple glissant. Ainsi, dans ces conditions, la ligne d'arbre aura une rotation chaotique si $s>s_{0}$ ce qui nécessite que la pulsation pendulaire soit du même ordre de grandeur que la pulsation d'alimentation car $m$ et $l$ sont des entiers de l'ordre de quelques unités et $c$ et $F$ sont comparables.

Si pour une machine synchrone où $c \ll F$ et $\omega_{0} \ll \omega$ ces conditions d'instabilité ne sont jamais réalisées par contre pour une machine à réluctance variable à vide, compte tenu de la faible inertie de ses disques (disques isolants avec peu de fer) il est possible de réaliser ces conditions d'instabilité comme nous allons le voir théoriquement dans l'exemple du prototype dont nous avons déjà présenté les essais et pour lequel $J=3,75 \mathrm{~kg} \cdot \mathrm{m}^{2}$ est faible.

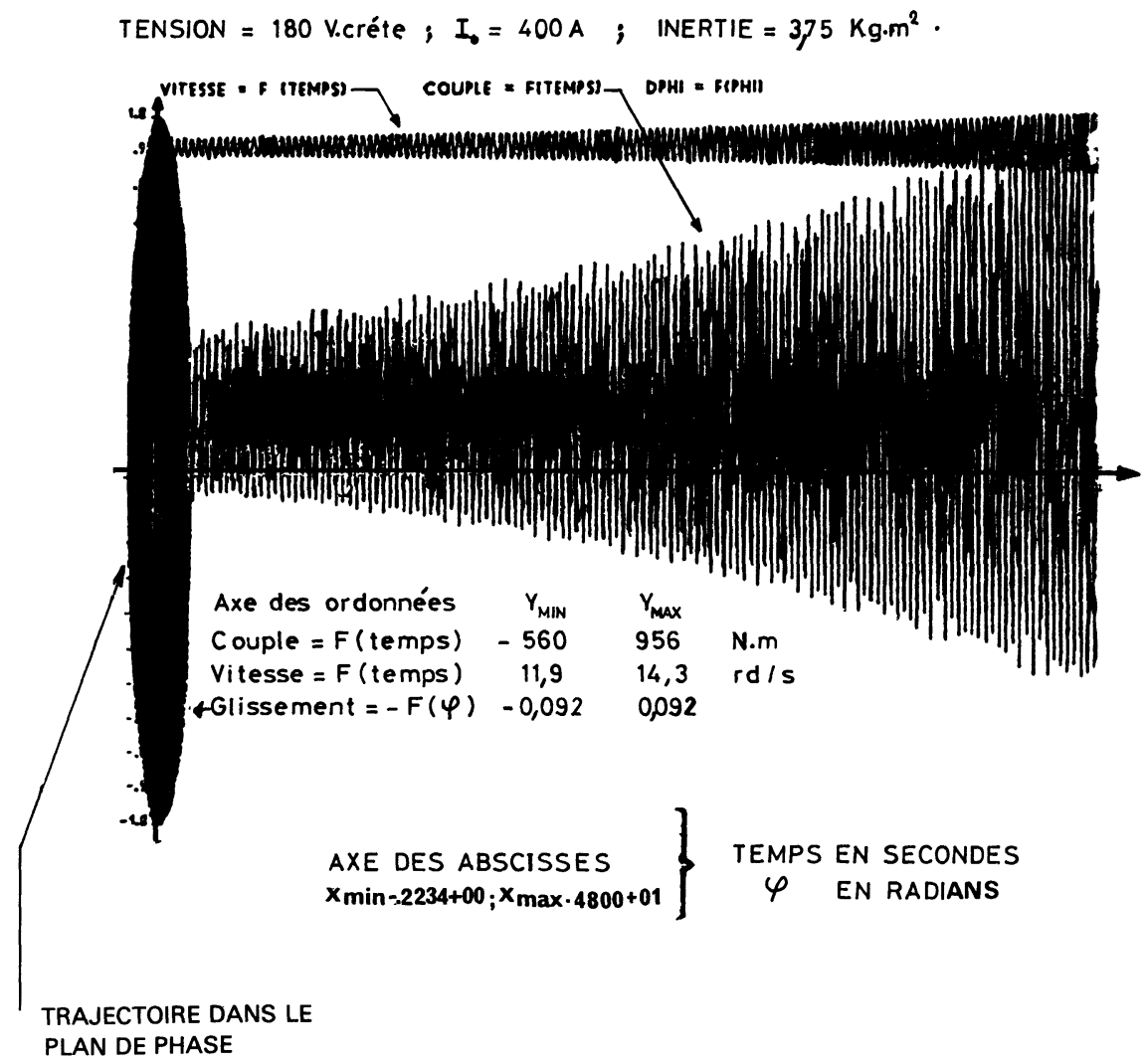

Fig. 18. - Simulation numérique d'un fonctionnement conduisant à une instabilité stochastique.

[Numerical simulation of a working with stochastic unstability.] 
Ainsi en considérant le couple glissant prépondérant de (25) pour lequel $m=2, l=3$ et $\gamma_{2,3}=-3 p r_{2} \phi K$ où $K \# N I_{0}$, nous obtenons l'expression suivante pour $s$ :

$$
s^{2}=\frac{8}{3} \frac{p^{2} V \sqrt{2}}{J \omega^{3}} \frac{N_{0}}{N} \frac{r_{1}}{r_{0}}\left(1+\sqrt{\frac{r_{2}}{r_{1}}}\right)^{2}
$$

ce qui nous conduit pour $V \sqrt{2}=180 \mathrm{~V}$ et $I_{0}=400 \mathrm{~A}$ à une valeur de 0,9 pour $s$ donc supérieure à $s_{0}=0,7$. L'expérimentation dans ces conditions n'étant pas possible car la machine à réluctance est accouplée au moteur du groupe Ward Léonard $\left(J \# 350 \mathrm{~kg} . \mathrm{m}^{2}\right)$, nous nous sommes limités à une simulation numérique de l'équation mécanique (30) avec les conditions d'instabilité précédentes. Nous avons alors effectivement constaté une instabilité qui n'existait pas en l'absence de couples parasites glissants. Ainsi comme on le voit sur la figure 18 , la trajectoire dans le plan de phase est une spirale divergente alors que des fluctuations de vitesse augmentent avec le temps.

\section{Conclusion.}

Cette étude présente les principaux résultats expérimentaux en fonctionnement moteur synchrone alimenté par le réseau triphasé $50 \mathrm{~Hz}$ acquis à ce jour sur la machine à réluctance variable à disques imbriqués ainsi que les résultats théoriques rendant compte du fonctionnement en régime linéaire. La concordance entre l'expérience et le modèle théorique est bonne. L'inertie des charges entraînées étant en général importante, nous pouvons également conclure que la stabilité d'une machine à réluctance variable alimentée directement par un réseau industriel est satisfaisante. L'étude et la réalisation de la machine ont été menées dans le cadre du contrat DRET 81/1032 « Moteur lent à réluctance variable ».

\section{Bibliographie}

[1] Rioux, C., "Théorie générale comparative des machines électriques établie à partir des équations du champ électromagnétique ». Rev. Gen. Elec. 79 (1970) no 5.

[2] Goyet, R., "Contribution à l'étude des machines à réluctance variable à disques imbriqués $»$, Thèse d'Etat, Paris 6, juin 1981.

[3] Salame, E., "Etude de l'accrochage et du fonctionnement en moteur de la machine à réluctance variable », stage de DEA ; Paris 6, juin 1981.

[4] Salame, E., «Etude des fonctionnements en moteur des machines à réluctance variable à disques imbriqués ", Thèse de Docteur-Ingénieur, Paris 6, mai 1983.

[5] Leveque, A., "Contribution à l'étude des machines à réluctance polydiscoïdes. Expérimentation en génératrice d'un prototype de $200 \mathrm{~kW}$ et modélisation à partir d'essais statiques ", Thèse de Docteur Ingénieur, Paris 6, septembre 1983.
[6] Bastos, J. P., Goyet, R., Lucidarme, J., Quichaud, G., Rioux-Damidau, F., " Performances of a multidisc variable reluctance machine », International Conference on Electrical Machines, Budapest, 6-7-8 septembre 1982 .

[7] Escande, F., DoveIL, F., « Renormalization method for computing the threshold of the large stochastic instability in two degrees of freedom Hamiltonian system », J. Stat. Phys. 26, no 2 (1981) 257-284.

[8] Croquette, V., Poitou, C., " Cascade de dédoublements de période et stochasticité à grande échelle des mouvements d'une boussole ». C.R. Hebd. Séan. Acad. Sci. Paris 292 (1982) 1353-1356.

[9] Croquette, V., "Déterminisme et chaos". Pour la Science, décembre 1982, p. 62-67.

[10] UnNewehr, L. E., КосH, W. H., " An axial air-gap reluctance motor for variable speed applications ", IEEE Trans. Power Appar. Syst. PAS 93, № 1, p. 367-374. 